\title{
Droplet and Aerosol Suspension Times in Ambient Air in Confined Spaces and Transmission of COVID-19: Influence of Environmental Factors
}

\author{
M. R. Islam, S. H. Naqib* \\ Department of Physics, University of Rajshahi, Rajshahi 6205, Bangladesh
}

Received 14 November 2020, accepted in final revised form 17 January 2021

\begin{abstract}
The COVID-19 pandemic, alternatively known as the coronavirus pandemic, is an unfolding pandemic of coronavirus disease 2019 (COVID-19) across the entire globe in an unprecedented proportion. COVID-19 is caused by severe acute respiratory syndrome coronavirus 2. The mode of transmission of COVID-19 is a subject of intense research. The airborne transmission is one prime possibility. Breathing and talking are natural processes which generate exhaled particles. The exhaled air is an aerosol/droplet composed of naturally produced particulates of varying size. The duration over which the aerosols/droplets are suspended in the air is an important factor. Long suspended aerosols/droplets are potential source of transmission, particularly in confined spaces. We have calculated times of suspension by considering various environmental factors, namely, the ambient temperature and relative humidity in a confined space, in this work. Both temperature and relative humidity affect the suspension time of the exhaled aerosols/droplets with varying degree. The effects of environmental factors are significant for aerosols, particularly for those with small radii. We have discussed the possible implications of our findings in this paper.
\end{abstract}

Keywords: COVID-19; Airborne transmission; Suspended aerosols and droplets; Effects of temperature and relative humidity.

(C) 2021 JSR Publications. ISSN: 2070-0237 (Print); 2070-0245 (Online). All rights reserved.

doi: http://dx.doi.org/10.3329/jsr.v13i2.50273 J. Sci. Res. 13 (2), 495-506 (2021)

\section{Introduction}

As of today (09 January, 2021), according to the https://www.worldometers.info/ coronavirus/ update, almost 90 million people all over the world have been infected by the novel coronavirus SARS-CoV-2. The death toll attributed to the COVID-19 has exceeded 1.9 million across the globe with no sign of the pandemic slowing down its pace of spread. In fact, the second wave, particularly in Europe and in the USA are gathering momentum in an unprecedented pace. From the early stage of this unfolding pandemic, it was thought that the environmental factors might have important role in determining the virus survivability and their transmission [1]. For example, relative humidity, defined as the ratio of water content to its saturation value in ambient air, as well as the ambient

\footnotetext{
*Corresponding author: salehnaqib@yahoo.com
} 
temperature, have been found to be strong environmental determinants of other viral transmissions like influenza [2,3]. It was suggested that hot and humid conditions do not favor the novel coronavirus and their transmission, yet this suggestion is still under considerable debate owing to many other uncertainties associated with the COVID-19 data [4]. Therefore, it is of paramount importance to understand properly the possible effects of environmental factors on the ongoing COVID-19 outbreak to support and facilitate necessary decision-making pertaining to the control of this disease.

The mode of transmission of novel coronavirus SARS-CoV-2 can be direct, indirect, via close contact with infected people (including asymptomatic and pre-symptomatic carriers) through secretions carrying viral load. These secretions include saliva and respiratory droplets which are expelled when an infected person sneezes, coughs, breaths, and even talks. In general, respiratory droplets are over 5-10 micron $(\mu)$ in size [5]; the size increases significantly during sneezing and coughing [6]. Larger droplets naturally contain larger viral load. Droplets with diameters $<5 \mu$ are known as droplet nuclei or aerosols. Such droplets have low viral load but can stay suspended in ambient air for a long time before settling on available surfaces due to combined effect of air flow and gravity. These long hanging particles in air can pose serious health hazard, particularly in a confined space [5].

In recent times, the World Health Organization (WHO) has issued health warning regarding the spread of COVID-19 by airborne transmission [5]. Categorically, airborne transmission can be defined as the spread of an infectious agent caused by the dissemination of aerosols which remains infectious when suspended in air over long distances and times. Airborne transmission of COVID-19 is particularly relevant in medical procedures which generate large volume of aerosols [5]. There is growing evidence that this particular mode of transmission is also active the absence of medical aerosol generating environments where natural process is the only source, especially in indoor settings with poor ventilation [5].

The dynamical processes involved with exhaled air and its flow have led to hypotheses regarding the possibility of aerosol transmission of SARS-CoV-2 [7-10]. Broadly speaking, the proposed scenarios suggest that respiratory droplets result in aerosols due to natural process of evaporation and normal breathing and talking creates aerosols. Therefore, a susceptible person could inhale the aerosol carrying viral load and could become infected with SARS-CoV-2. However, the amount of infectious dose of viable SARS-CoV-2 required to infect a person is not clear at this moment [11]. There are subjective elements involved with this factor, like the state of health and level of immunity of the susceptible subject, and requires further investigation for a clearer picture to appear. There are several recent studies concerned with generation of droplets and aerosols during exhalation, talking, coughing, and sneezing [12-14]. It has been suggested that there is high variability among individuals in terms of droplet/aerosol emission rates during talking and with increased rates linked with raised level of vocalization [9].

There are several reports on suspension time and dynamics of respiratory droplets and aerosols in the existing literature. Most of these studies are experimental in nature or 
computer simulations [15,16]. In a recent work, Singh and Kaur [17] calculated the suspension time of aerosols with different diameters by using the Newtonian-Stokes formalism. The possible environmental effects due to variation of temperature and humidity were not considered. The environmental factors are thought to play important roles in the transmission of COVID-19 [16]. It has been reported that high temperature and high relative humidity disrupt SARS-CoV-2 viability and activation [4]. The precise reasons though are still not clear [18-20]. Considering the available information, Morawska and Cao [10] stressed that small particles with viral content may travel in indoor, covering distances reaching 10 meters starting from the point of emission, thereby activating aerosol transmission. Recently, Paules et al. [21] pointed out that the airborne transmission may also occur besides close distance contact mode. Both experimental and computational fluid dynamic approaches support such assumptions. Asadi et al. [14] paid experimental attention to the emitted particle number and to their size distribution of aerosol emissions occurring during human speech and found a high variability among individuals. Analytical studies taking into consideration of the prime environmental factors - temperature and humidity, on the droplet and aerosol suspension times in the ambient air for possible airborne transmission of the SARS-CoV-2 are very limited.

Against this backdrop, we feel that an analytical theoretical study of the droplet and aerosol suspension times for different sizes under different environmental factors is important. Our focus, in this study, is on pre-symptomatic asymptomatic virus carriers, i.e., subjects who do not show noticeable pathological symptoms like coughing and sneezing. Such subjects do not emit high velocity particles in the environment such as those which happens while coughing and particularly while sneezing. We aim to carry out theoretical calculations of droplet and aerosol suspension times under different temperature and relative humidity in this paper in case of low velocity emission in confined spaces. The rest of the paper is structured as follows. Section 2 presents the calculational details and results. We discuss the implications of our findings and summarize the conclusions in Section 3.

\section{Model, Calculations, and Results}

What happens to the emitted droplets and aerosols (or particles to mean both) during exhalation, talking, coughing, or sneezing in ambient air? The emitted particles have a distribution of velocities and behave as small projectiles in air. These velocities diminish rapidly due to the viscosity of air and after a very short while gravity becomes the major factor in controlling the motion of the larger and heavier droplets. The motion and state of the smaller particles can be affected by the flow of air. Herein, we have omitted the effect of air flow and possible occurrence of turbulence in our calculations. We have also neglected the effect of buoyancy on the falling particulates due to air. This is reasonable [22] because of the almost three orders of magnitude difference between the density of exhaled substances (largely water based) and that of the ambient air. It should be stressed that the calculations to follow are more appropriate for indoor environment. 
Gravity is ubiquitous. Under the influence of gravity, the emitted particles are accelerated downwards towards the ground. Air, on the other hand, is a viscous fluid and moving particles through air feels viscous drag opposing the acceleration due to gravity. As a result, the particle reaches a terminal velocity. The magnitude of this limiting velocity which remains unchanged till the particle settles on the ground depends on the size of the particle and viscosity of the fluid through which it moves.

In this study we model the situation where an adult is the emitter of particles during exhalation. We take the mean heights of adult men and women are approximately 164 and $178 \mathrm{~cm}$, respectively [23]. The average of the means is $171 \mathrm{~cm}$. The sources of emission (nose and mouth) of droplets and aerosols lie approximately $15 \mathrm{~cm}$ below the top of the skull. Therefore, the effective height above the ground has been considered as $156 \mathrm{~cm}$. For macroscopic bodies, released from moderate heights $(\sim 10 \mathrm{~m})$, the viscous drag resisting the motion towards ground is often neglected. The fall down time, $t_{\mathrm{f}}$, is independent of the horizontal component of the velocity of the macroscopic body, if any, and is given by, $t_{\mathrm{f}}=(2 \mathrm{~h} / \mathrm{g})^{1 / 2}$, independent of the mass and size of the falling object. Here, $h$ is the height of release and $g$ is the acceleration due to gravity. For $h=1.56 \mathrm{~m}, t_{\mathrm{f}}=0.564$ $\mathrm{s}$ with standard value of $g=9.81 \mathrm{~m} / \mathrm{s}^{2}$. This situation changes drastically for small particulates. The equation motion for the emitted particulates of radius $r$ during exhalation falling towards the ground can be expressed as,

$$
m \frac{d v(t)}{d t}=m g-(6 \pi \eta r v(t))
$$

In the above differential equation, $m$ is the mass of the particle, $v(t)$ is the time dependent velocity of descent, and $\eta$ is the coefficient of viscosity of ambient air through which the droplet/aerosol falls down. The second term within the bracket in the right-hand side is the force due to viscous drag which opposes the force due to the acceleration due to gravity. This force due to viscous drag slows down the velocity of descent which otherwise would have increased uniformly under the influence of gravity. Eqn. 1 is a simple first-order differential equation, where the two variables $t$ and $v(\mathrm{t})$ can be separated. The general solution of Eqn. 1 is given by,

$v(t)=\operatorname{Cexp}\left(-\frac{t}{t_{0}}\right)+t_{0} g$

In this equation $C$ is a constant and $t_{0}$ is a characteristic time which can the termed as the time constant that determines the rate of exponential decrease in the velocity of descent. For this particular problem, $t_{0}=(m / 6 \pi \eta r)$. The constant, $C$ can be obtained by using the boundary condition, $v(t)=0$ at $t=0$ in Eqn. 2, giving $C=-g t_{0}$. Substituting this value of $C$ into Eqn. 1, we get,

$v(t)=g t_{0}\left[1-\exp \left(-\frac{t}{t_{0}}\right)\right]$

The velocity of descent, $v(\mathrm{t})$ at any instant of time $t$ is related to the height from the ground at that particular instant $h(t)$ by the equation, 
$v(t)=\frac{d h(t)}{d t}$

Therefore, one can obtain $h(t)$ (measured from the point of emission) by integrating the above equation with respect to time. Using Eqn. 3 and the boundary condition $h(t)=0$, at $t$ $=0$, we obtain,

$h(t)=g t_{0}\left[t+t_{0}\left\{\exp \left(-\frac{t}{t_{0}}\right)-1\right\}\right]$

The time of suspension or the fall down time or the total time of descent, has the same meaning in this investigation. This time, $t_{\mathrm{s}}$, is the time required for a droplet/aerosol to reach the ground which originated at a height $h$ from the ground at a time $t=0$. In all subsequent calculations, we take $h=1.56 \mathrm{~m}$. Furthermore, it should be noted that the expression for the time constant involve the mass of the particulate, $m$. It is possible to express this mass in terms of the volume and density of the droplet/aerosol. To minimize the surface energy, droplets and aerosols assume spherical shape. The density, $\rho$, of novel SARS-CoV-2 is not known precisely. Therefore, a reasonable approximation should be the density of water, taken as $1 \mathrm{~g} / \mathrm{cm}^{3}$ (in the CGS unit) or $1000 \mathrm{~kg} / \mathrm{m}^{3}$ (in the SI unit). With these assumptions, the expression for $t_{0}$ becomes, $t_{0}=\frac{2}{9}\left(\frac{\rho r^{2}}{\eta}\right)$. The temperature dependence of the density has been neglected. For temperatures of interest $\left(10^{\circ} \mathrm{C}\right.$ to $50^{\circ} \mathrm{C}$ ), this is reasonable because the variation in the density of water with temperature is minute compared to the variation of the coefficient of viscosity.

The common environmental factors are pressure, temperature, and humidity. Among these, pressure has a little effect on the viscosity of gases. In fact, for ideal gases, there is no effect of pressure on viscosity. Real gases, at around the atmospheric pressure, behave quite closely as ideal gases. Thus, in our calculations, we have not considered the effect of atmospheric pressure on the time of suspension, $t_{\mathrm{s}}$. It is also worth noting that compared to temperature and humidity the range over which atmospheric pressure varies is quite small. Both temperature and humidity affect the coefficient of viscosity. Unfortunately, accurate and analytical expressions considering the real intermolecular potential to describe the effect of temperature and humidity on the viscosity of air over wide ranges of parameter values do not exist in the literature. Under the circumstances, we have used the experimentally determined temperature and humidity dependent values of the viscosity of air to calculate $t_{\mathrm{s}}$ as functions of temperature and humidity [24].

We start by showing the size (radius) dependence of the droplet/aerosol on the time of suspension in Fig. 1 below. Here, the value of viscosity, $\eta$, has been taken as $1.85 \times 10^{5}$ $\mathrm{kg} / \mathrm{m}$-s at standard temperature and relative humidity $\left(25^{\circ} \mathrm{C}\right.$ and $50 \%$, respectively). 


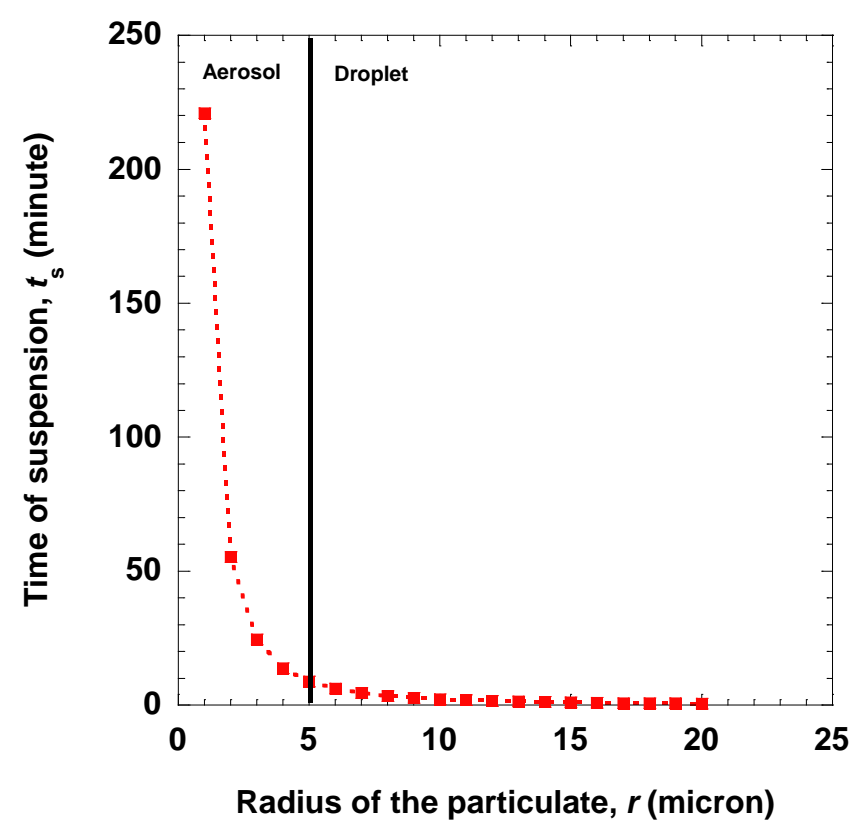

Fig. 1. Time of suspension versus the radius of the exhaled particulate. The full vertical line separates the aerosols from the droplets.

Fig. 1 illustrates some interesting features. Initially, the time of suspension decreases rapidly with increasing radius of the exhaled particulates. For example, $t_{\mathrm{s}} \mathrm{s}$ for aerosols of radii $1 \mu$ and $5 \mu$ are $221 \mathrm{~min}(3 \mathrm{~h}$ and $41 \mathrm{~min}$ ) and $8.83 \mathrm{~min}$, respectively. For droplets, on the other hand, the times of suspension are $2.21 \mathrm{~min}$ and $0.56 \mathrm{~min}$ for radii of $10 \mu$ and 20 $\mu$, respectively. This shows that the time over which exhaled particulates hang in air varies over wide range depending on the size. For droplets with radii over $15 \mu, t_{\mathrm{s}}$ become less than a min. This implies that for $r>15 \mu$, possibility of airborne transmission is low, particularly in confined spaces. Large droplets settle quite quickly to the ground and the possibility of airborne transmission is diminished. Such droplet can contribute to fomite transmission which is a proven mode of transmission of SARS-CoV-2 [25-27].

Next, we have displayed the effect of temperature on $t_{\mathrm{s}}$ at standard relative humidity (RH) in Fig. 2. The temperature dependent values of viscosity of air have been taken from previously published work [23]. 


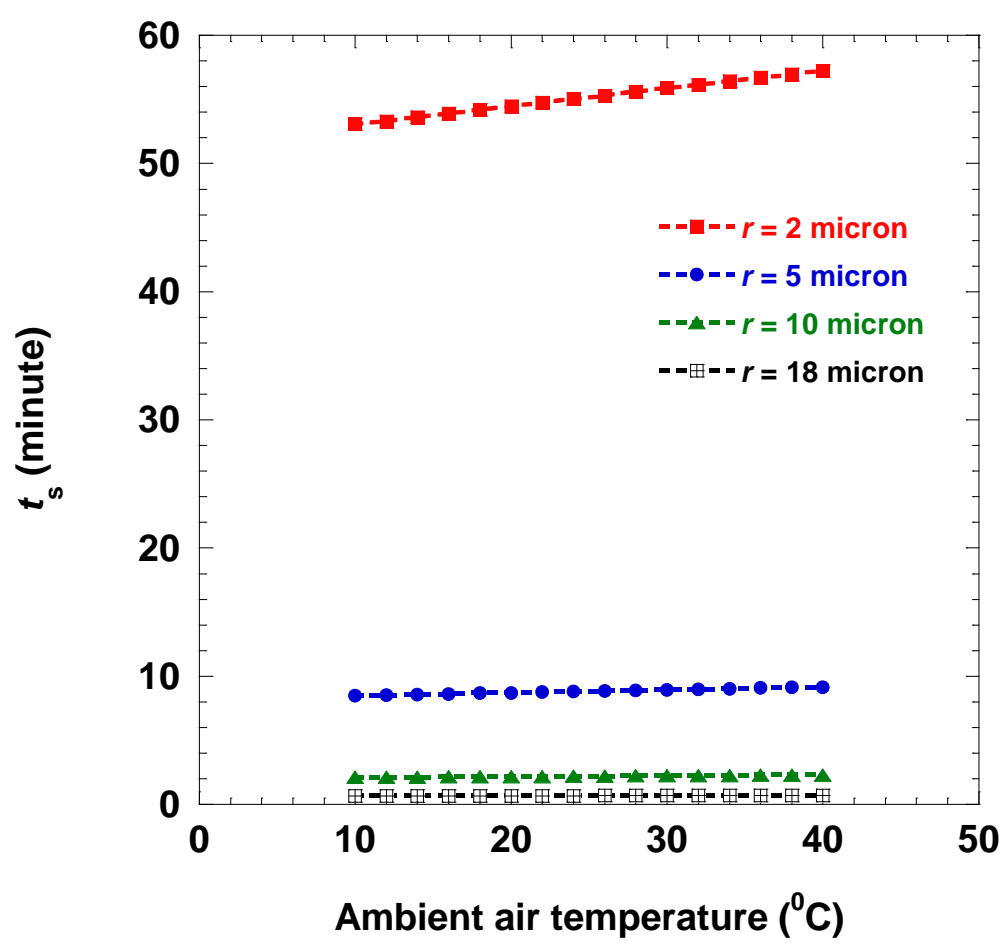

Fig. 2. Time of suspension versus ambient air temperature for different radii of the exhaled aerosols and droplets at a fixed value of $\mathrm{RH}$.

Fig. 2 discloses that temperature has significant effect on $t_{\mathrm{s}}$ only for small aerosols. For example, the $t_{\mathrm{s}}$ is about $53 \mathrm{~min}$ at $10^{\circ} \mathrm{C}$ for an aerosol of radius $2 \mu$ which increases to $57.25 \mathrm{~min}$ at $40^{\circ} \mathrm{C}$. For droplets, the time of suspension does not depend significantly on temperatures of interest.

The effect of relative humidity on $t_{\mathrm{s}}$ is displayed in Fig. 3. Here we have shown results only for the particulates in the form of aerosols. The effect of $\mathrm{RH}$ on $t_{\mathrm{s}}$ for droplets $(r>5 \mu)$ is insignificant.

It is seen from Fig. 3 that RH affects the time of suspension of aerosols which in turn depends on the temperature of the ambient air. The effect of RH on $t_{\mathrm{s}}$ becomes significant at high temperatures. 

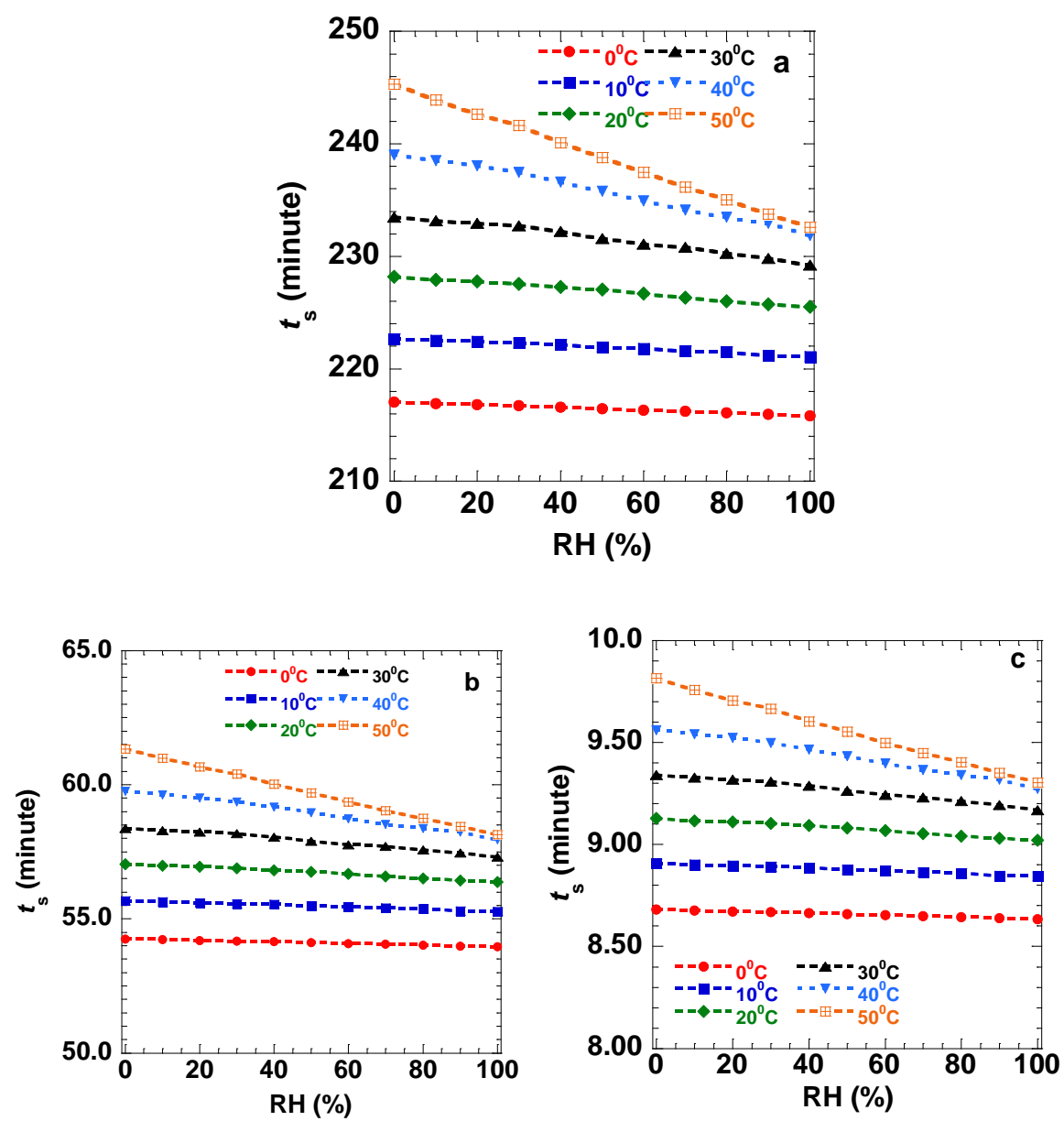

Fig. 3. Effect of relative humidity on $t_{\mathrm{s}}$ at different temperatures for (a) $r=1 \mu$, (b) $r=2 \mu$, and (c) $r$ $=5 \mu$.

\section{Discussion and Conclusion}

Our calculations demonstrate clearly that environmental parameters affect the time of descent with varying degrees. The major effect on $t_{\mathrm{s}}$ comes from the size of the exhaled particulates. This is particularly true for small aerosols. Particles with radii over $5 \mu$ fall down to the ground within minutes or a few tens of seconds. Calculations of $t_{\mathrm{s}} \mathrm{s}$ how that only the aerosols hang in the air for long. Aerosols of radii $\sim 1 \mu$ are suspended in air for hours. The viral loads in such small particulates are expected to be low but still might be enough to infect people with conditions which suppress natural immunity. The droplets, on the other hand settle quite quickly to the ground or on structures (for example, furniture) below the point of emission. In this case, respiratory secretions or droplets exhaled by infected individuals can contaminate surrounding surfaces and objects, 
creating fomites (contaminated surfaces). Viable SARS-CoV-2 virus and/or RNA detected by RT-PCR can be found on those surfaces for periods ranging from hours to days [25], depending on the ambient environment (including temperature and humidity) and the type of surface [28,29]. Thus, transmission of COVID-19 may also occur indirectly through touching such surfaces or objects contaminated with virion from an infected individual. Therefore, larger droplets pose a risk of different kind from that due to the airborne transmission mode.

Beside the size of the aerosols/droplets, both temperature and $\mathrm{RH}$ affect $t_{\mathrm{s}}$. For a fixed $\mathrm{RH}, t_{\mathrm{s}}$ increases with increasing temperature. The rate of increment though is strongly size dependent. For droplets the effect of temperature on $t_{\mathrm{s}}$ is insignificant. For small aerosols, $t_{\mathrm{s}}$ increases notably with increasing temperature (Fig. 2). The overall physical effect of temperature on airborne and other modes of transmission in indoor environment is quite complicated. Because an increase in $t_{\mathrm{s}}$ with temperature for aerosols implies greater risk of infection by inhalation for an uninfected subject, but increase of temperature also aids evaporation and drying of the exhaled particulates which will decrease the viral load in individual droplets.

RH has an opposite effect compared to that due to temperature. Increase in RH leads to a decrease in the $t_{\mathrm{s}}$. The rate of decrement depends strongly on the temperature of the ambient air. At low temperature the variation in RH changes $t_{\mathrm{s}}$ very weakly. The effect becomes significant at high temperatures (Figs. 3), irrespective of the size of the aerosol/droplet. For example, at $40^{\circ} \mathrm{C}, t_{\mathrm{s}}=239 \mathrm{~min}$ with $\mathrm{RH}=0 \%$ for an aerosol with $r=$ $1 \mu, t_{\mathrm{s}}$ decreases to $232 \mathrm{~min}$ for the same aerosol at the same temperature with $\mathrm{RH}=$ $100 \%$. The situation at $50^{\circ} \mathrm{C}$ is as follows: $t_{\mathrm{s}}=245 \mathrm{~min}$ with $\mathrm{RH}=0 \%$ for an aerosol with $r=1 \mu, t_{\mathrm{s}}$ decreases to $233 \mathrm{~min}$ for the same aerosol at the same temperature with $\mathrm{RH}=$ $100 \%$. It is interesting to note that, for $100 \% \mathrm{RH}$ the difference between $t_{\mathrm{S}} \mathrm{s}$ for $40^{\circ}$ and $50^{\circ} \mathrm{C}$ is almost zero. Therefore, one can conclude that at high temperatures with high $\mathrm{RH}$ $t_{\mathrm{s}}$ is affected little by the variation of temperature. The overall physical effect of relative humidity on the risk of airborne and other modes of transmission is also difficult to assess. This is because, even though $t_{\mathrm{s}}$ decreases significantly with increase in the RH at high temperatures, an increase in the RH decreases the rate of evaporation and drying time of larger droplets.

It should be noted that the rate of evaporation of droplets and aerosols depend on ambient temperature, RH and natural and forced convections [30, 31]. The effect of forced convection due to free flow of wind is minimal in confined spaces. Nevertheless, evaporation is a spontaneous process due to Maxwellian velocity distribution of the kinetic energies of the molecules at any temperature and RH. The effect of surface tension is also important. The surface area to volume ratio is extremely high in aerosols. The total energy of such small particles is significantly dominated by surface energy. Once all these effects are considered, the analyticity of the mathematical treatment regarding evaporation is lost [30, 31]. In this study, our focus has been on a simple analytic methodology to estimate the droplet/aerosol suspension time in air in confined spaces. Still, we have tried to estimate roughly the effect of evaporation on droplet suspension time in air under 
standard atmospheric conditions following the methodology developed by Pinheiro and Vedovodo [30]. For large droplets (with sub-millimeter radius) the effect of mass-loss due to evaporation is small. For example, the rate of mass-loss for a droplet of $r=0.3 \mathrm{~mm}$ is $1 \% / \mathrm{s}$ consistent with the findings in Ref. [31]. Such large droplets fall very quickly to the ground and therefore, evaporation does not play any significant role as far as possibility of airborne transmission is concerned. The situation for smaller droplets and aerosols is different. In the presence of evaporation, rate of loss of mass is rapid, viral loads are dispersed easily and the suspension time in the air increases significantly.

There are of course biological effects of temperature and $\mathrm{RH}$ on the virus itself. In this investigation only the physical motion of the aerosols/droplets are considered.

In our calculation of $t_{\mathrm{s}}$, we have omitted any effect due to turbulence and also the initial speed distribution of the exhaled particulates. In indoor environments nonlinear fluid dynamics is not very relevant. Average speed of exhaled particulates during nasal breathing is quite small, $\sim 1.4 \mathrm{~m} / \mathrm{s}$ [17]. Inclusion of such small initial velocity will not affect the calculations presented herein in any significant way.

It is seen that size of the exhaled particulates is the most important factor in determining $t_{\mathrm{s}}$. It is reasonable to assume that larger aerosols/droplets contain larger viral load. But quite counterintuitively, it may happen that small aerosols are more dangerous for possible infection [6]. Aerosols $(r<5 \mu)$ suspended in air might carry a higher concentration of virus compared to that in larger droplets [32-34]. Moreover, large droplets have less chance to penetrate deeply in the respiratory system and there is possibility that those might be deactivated by the effective first structural and defense barrier of the mucosa [35]. On the other hand, aerosolised virus hangs in the air for a very long time compared to droplets, and can be transmitted airborne through inhalation deep into the lungs [36,37], by passing the natural defenses of the upper respiratory system.

The time over which the exhaled particles hang in the air is an important parameter to assess the risk of airborne transmission of COVID-19. In this paper it has been demonstrated that the time of suspension depends both on temperature and relative humidity. The effects are intermingled and depend strongly on the size of exhaled particulates. The calculations presented in this study are relevant to indoor environments like those in confined public offices, ticket counters, queues in the bank etc. In such places, pre-symptomatic or asymptomatic subjects can emit substantial viral load through normal talking and nasal breathing. Public health policymakers should take the case of airborne transmission seriously. This particular mode has the potential to become very effective in transmitting the SARS-CoV-2in indoor environments. We hope that this work will help the policymakers to formulate guidelines to manage public health situations in indoor environments and confined spaces.

\section{Data availability}

The data sets generated and/or analyzed in this study are available from the corresponding author on reasonable request. 


\section{References}

1. D. J. Prata, W. Rodriguse, and P. H. Bermejo, Sci. Total Environ. 729, ID 138862 (2020). https://doi.org/10.1016/j.scitotenv.2020.138862

2. A. I. Barreca and J. P. Shimshack, Am. J. Epidemiol. 176, 114 (2012). https://doi.org/10.1093/aje/kws259

3. J. Shaman, E. Goldstein, and M. Lipsitch, Am. J. Epidemiol. 173, 127 (2011). https://doi.org/10.1093/aje/kwq347

4. Y. Ashkenazy, E. Asher, S. Havlin, and A. Sela, Optimal COVID-19 Infection Spread under Low Temperature, Dry Air, and Low UV Radiation (2020). https://arxiv.org/pdf/2007.09607.pdf.

5. World Health Organization (WHO), Transmission of SARS-CoV-2: Implications for Infection Prevention Precautions (2020).

6. I. M. Viola, B. Peterson, G. Pisetta, G. Pavar, H. Akhtar, E. Menoloascina, E. Mangano, K. E. Dunn, R. Gabl, A. Nila, E. Molinari, C. Cummins, G. Thompson, C. M. McDougall, T. Y. M. Lo, F. C. Denison, P. Digard, O. Malik, M. J. G. Dunn, and F. V. Mehendale, Facecovering, Aerosol Dispersion and Mitigation of Virus Transition (2020). https://arxiv.org/ftp/arxiv/papers/2005/2005.10720.pdf

7. R. Mithal, R. Ni, J. -H. Seo, J. Fluid Mech. 894 (2020). https://doi.org/10.1017/jfm.2020.330

8. L. Bourouiba, JAMA 323, 1837 (2020). https://doi.org/10.1001/jama.2019.13239

9. S. Asadi, N. Bouvier, A. S. Wexler, and W. D. Ristenpart, Aerosol Sci. Technol. 54, 635 (2020). https://doi.org/10.1080/02786826.2020.1749229

10. L. Morawska and J. Cao, Environ. Int. 139, 105730 (2020). https://doi.org/10.1016/j.envint.2020.105730

11. J. Gralton, T. R. Tovey, M-L. McLaws, and W. D. Rawlinson, J. Med. Virol. 85, 2151 (2013). https://doi.org/10.1002/jmv.23698

12. V. Standnytskyi, C. E. Bax, A. Bax, and P. Anfinrud, Proc. Ntl. Acad. Sci. 117, 11875 (2020). https://doi.org/10.1073/pnas.2006874117

13. G. A. Somsen, R. C. Van, S. Kooij, R. A. Bem, and D. Bonn, Lancet Respir. Med. S2213260020302459 (2020).

14. S. Asadi, A. S. Wexler, C. D. Cappa, S. Barreda, N. M. Bouvier, and W. D. Ristenpart, Sci. Rep. 9, 2348 (2019). https://doi.org/10.1038/s41598-019-38808-z

15. M. J. Evan, Avoiding COVID-19: Aerosol Guidelines (2020). https://doi.org/10.1101/2020.05.21.20108894

16. C. Poirier, W. Luo, M. S. Majumder, D. Liu, K. Mandl, T. A. Mooring, and M. Santillana, SSRN (2020). http://dx.doi.org/10.2139/ssrn.3552677

17. N. Singh and M. Kaur, On the Airborne Aspect of COVID-19 Coronavirus (2020). https://arxiv.org/pdf/2004.10082.pdf

18. K. Chan, J. Peiris, S. Lam, L. Poon, K. Yuen, and W. Seto, Adv. Virol. 2011, ID 734690 (2011). https://doi.org/10.1155/2011/734690

19. E. F. Foxman, J. A. Storer, M. E. Fitzgerald, B. R. Wasik, L. Hou, H. Zhao, P. E. Turner, A. M. Pyle, and A. Iwasakiet, Proc. Ntl. Acad. Sci. 112, 827 (2015). https://doi.org/10.1073/pnas.1411030112

20. J. J. Cannell, R. Vieth, J. C. Umhau, M. F. Holick, W. B. Grant, S. Madronich, C. F. Garland, and E. Giovannucci, Epidemiology Infection 134, 1129 (2006). https://doi.org/10.1017/S0950268806007175

21. C. I. Paules, H. D. Marston, and A.S. Fauci, JAMA 323, 707 (2020). https://doi.org/10.1001/jama.2020.0757

22. S. Xu, J. Cui, and X. Ren, $2^{\text {nd }}$ Int. Conf. on Electronic and Mechanical Engineering and Information Technology (EMEIT-2012) (Atlantis Press, Paris, France, 0482, 2012).

23. A. Jelenkovic, R. Sund, Y. Yokoyama, et al., Sci. Rep. 6, ID 28496 (2016). https://www.nature.com/articles/srep28496 
24. M. Boukhriss, K. Zhani, and R. Ghribi, Desalination Water Treatment 51:4-6, 1290 (2013). https://doi.org/10.1080/19443994.2012.714925

25. N. V. Doremalen, T. Bushmaker, D. H. Morris, M. G. Holbrook, et al., New Engl. J. Med. 382, 1564 (2020). https://doi.org/10.1056/NEJMc2004973

26. P. Y. Chia, K. K. Coleman, Y. K. Tan, M. Gum, et al., Nat. Comm. 11, 2800 (2020).

27. B. Pastorino, F. Touret, M. Gilles, X. D. Lamballerie, and R. N. Charrel, Emerg. Infect. Dis. 26, (2020). https://doi.org/10.3201/eid2609.202267

28. Z. -D. Guo, Z. -Y. Wang, S. -F. Zhang, X. Li, L. Li, C. Li, et al., Emerg. Infect. Dis. 26, 1583 (2020). https://doi.org/10.3201/eid2607.200885

29. M. J. Matson, C. K. Yinda, S. N. Seifert, T. Bushmaker, R. J. Fischer, N. van Doremalen, et al., Emerg. Infect. Dis. 26, ID 9 (2020). https://doi.org/10.3201/eid2609.202267

30. Abgail P. Pinheiro, Joao Marcelo Vedovodo, Flow, Turbulence Combust. 102, 537 (2019). https://doi.org/10.1007/s10494-018-9973-8

31. D. C. Kincaid, T. S. Longley, Transact. ASAE. 32, 457 (1989). https://doi.org/10.13031/2013.31092

32. D. K. Milton, M. Patricia Fabian, B. J. Cowling, M. L. Grantham, and J. J. McDevitt, PLoS Pathogens 9, ID e1003205 (2013). https://doi.org/10.1371/journal.ppat.1003205

33. W. G. Lindsley, J. D. Noti, F. M. Blachere, R. E. Thewlis, S. B. Martin, S. Othumpangat, B. Noorbakhsh, et al., J. Occupat. Environ. Hygiene 12, 107 (2015). https://doi.org/10.1080/15459624.2014.973113

34. Leung, H. L. Nancy, Chu, K. W. Daniel, Shiu, Y. C. Eunice, J. J. McDevitt, Hau, J. P. Benien, Y. Li, D. K. Milton, et al., Nature Medicine 26, 676 (2020). https://doi.org/10.1038/s41591-020-0843-2

35. W. J. Fokkens and R. A. Scheeren, Paediatric Respir. Rev. 1, 336 (2000). https://doi.org/10.1053/prrv.2000.0073

36. G. Zayas, M. C. Chiang, E. Wong, F. MacDonald, C. F. Lange, A. Senthilselvan, and M. King, BMC Pulmonary Medicine 12, 11 (2012). https://doi.org/10.1186/1471-2466-12-11

37. J. Yan, M. Grantham, J. Pantelic, P. J. B. D. Mesquita, B. Albert, F. Liu, S. Ehrman, D. K. Milton, and E. Consortium, Proc. Ntl. Acad. Sci. 115, 1081 (2018). https://doi.org/10.1073/pnas.1716561115 